\title{
Chirped Optical Solitons: High Degree Pulse Compression
}

\author{
K. Senthilnathan, ${ }^{1}$ K. Nakkeeran, ${ }^{2}$ Qian $\mathrm{Li}^{3}$ and P. K. A. Wai ${ }^{3}$ \\ ${ }^{1}$ Department of Physics, National Institute of Technology, Rourkela, Orissa, 769 008, India \\ ${ }^{2}$ School of Engineering, Fraser Noble Building, King's college, University of Aberdeen, Aberdeen AB24 3UE, UK. \\ ${ }^{3}$ Photonics Research Center and Department of Electronic and Information Engineering, The Hong Kong Polytechnic \\ University, Hong Kong. Phone: +852-27666231, Fax:+852-23628439, Email:enwai@polyu.edu.hk
}

\begin{abstract}
We propose the high degree pulse compressor based on the chirped higher order soliton pulses in the exponentially decreasing dispersion.
\end{abstract}

\section{Introduction}

In optical fibers, a dynamical balance occurs between the linear effect, anomalous group velocity dispersion (AGVD) which produces negative chirp, and nonlinear effect, self-phase modulation (SPM) which generates positive chirp. The resulting pulse exhibits zero chirp, and is called a soliton [1]. Soliton pulse propagation in optical fibers is governed by the well-known nonlinear Schrödinger equation (NLSE) [1]. It is obvious that an ideal fundamental soliton is robust. However, the pulse propagation in real optical fiber systems leads to serious limitations on the design of the communication system. Fiber loss is found to be most harmful one. The loss compensation techniques have been proposed with amplifiers [1]. Another interesting approach has also been suggested to use the exponentially decreasing dispersion [1]. For the first time, the fabrication of 38$\mathrm{km}$ of exponentially dispersion decreasing fiber was produced to compensate the fiber loss [1]. Thus, in the modern technology, the desired exponentially dispersion decreasing fiber can easily be fabricated by tapering the core of the optical fiber. However, in this paper, we obtain the exponential decreasing dispersion as a condition for the soliton pulse propagation. Contrary to the above studies, we investigate the existence of chirped soliton and use it for achieving the effective pulse compression.

It is well known that soliton pulse compression and adiabatic pulse compression techniques could not satisfy all the requirements since both techniques generate considerable amount of undesired pedestal [1]. It has been demonstrated that increasing the input soliton order enhances the compression factor but the compressed pulses suffer from the pedestal as the higher order solitons do not have linear chirp. Therefore, it is of great interest to develop a compression technique capable of achieving both high quality compression and large compression factor.

Instead of the chirp free solitons, more recently, attention has been riveted on the generation of chirped solitons. It has been suggested that chirped solitary waves could be compressed more efficiently if the dispersion decreases approximately exponentially [2]. Recently, self-similar analysis has been used to obtain the linearly chirped solitary waves [3]. Very recently, using the self-similar analysis, chirped Bragg solitary pulses have been theoretically generated near the photonic bandgap of a non-uniform fiber Bragg grating, and the possibility of pedestal free Bragg soliton pulse compression is examined [4]. Very recently, we have clearly explained the formation of chirped soliton in the exponentially dispersion decreasing media using the variational analysis and theoretically generated the chirped soliton by Hirota-bilinear method. This chirped soliton has been utilized to achieve efficient chirp and pedestal free pulse compression [5].

The main theme of this paper is to achieve the high degree of pulse compression by means of chirped higher order solitons. In fact, the high degree pulse compression will be achieved through the special chirped soliton breather pulse, whose amplitude is twice of that of the fundamental soliton, which can be obtained from the general higher order solitons.

\section{Theoretical Model}

Pulse propagation in dispersion varying nonlinear media is governed by the NLSE

$i \frac{\partial \Psi}{\partial z}-\frac{\beta(z)}{2} \frac{\partial^{2} \Psi}{\partial t^{2}}+\gamma|\Psi|^{2} \Psi=0$,

where $\psi(z, t)$ is the slowly varying envelop of the optical pulse, $\beta(z)$ is the dispersion varying profile, $\gamma$ is the cubic nonlinear coefficient, $z$ is the distance along the fiber, and $t$ is time. To check the possibility of soliton pulse propagation of Eq. (1), we apply the well known Painlevé analysis. The Painlevé analysis implies that the dispersion must vary in an exponential manner (and/or the dispersion should be constant) for the system Eq. (1) to be completely integrable i.e., $\beta=\beta_{0} \exp (-\sigma z)$ where $\beta_{0}$ and $\sigma$ are integration constants. It should be emphasized that the exponential scaling (for the dispersion profile), obtained by Painlevé analysis, is the same as that obtained through self-similar analysis [3, 4]. In the exponentially dispersion decreasing media, the chirped optical solitons are formed basically because of the growth of the chirp in contrast to a zero chirp in case of the conventional solitons [5].

\section{Chirped Higher Order Solitons}

We now demonstrate the pulse compression using higher order soliton property as well as chirped soliton concept to achieve the high degree compression. That is, we investigate the pulse compression by a special pulse whose amplitude is doubled compared to that of the 
fundamental soliton, also known as chirped soliton breather. The continuous interaction of the $N$ solitons gives rise to the periodic behavior of the total pulse. We theoretically generate the chirped two soliton by means of Bäcklund transformation and the same is given by

$\Psi=\sqrt{\frac{-\beta_{0}}{\gamma}} e^{\alpha_{0} \beta_{0} z / 2} \frac{F_{0}}{G_{0}}$, where,

$F_{0}=\left(\tau_{2}^{2}-\tau_{1}^{2}\right)\left[\tau_{2} e^{i \theta_{1}} \operatorname{sech}\left(\xi_{1}\right)-\tau_{1} e^{i \theta_{2}} \operatorname{sech}\left(\xi_{2}\right)\right] e^{i m}$,

$G_{0}=\tau_{1}^{2}+\tau_{2}^{2}-2 \tau_{1} \tau_{2}\left[\begin{array}{l}\tanh \left(\xi_{1}\right) \tanh \left(\xi_{2}\right) \\ +\sec h\left(\xi_{1}\right) \sec h\left(\xi_{2}\right) \cos \left(\theta_{2}-\theta_{1}\right)\end{array}\right]$,

$\xi_{1}=\frac{t e^{\alpha_{0} \beta_{0} z}}{\tau_{1}}, \xi_{2}=\frac{t e^{\alpha_{0} \beta_{0} z}}{\tau_{2}}, \theta_{1}=-\frac{e^{\alpha_{0} \beta_{0} z}}{2 \alpha_{0} \tau_{1}^{2}}, \theta_{2}=-\frac{e^{\alpha_{0} \beta_{0} z}}{2 \alpha_{0} \tau_{2}^{2}} \quad$ and

$m=\frac{\alpha_{0} t^{2} e^{\alpha_{0} \beta_{0} z}}{2}$.

Here the physical parameters $\tau_{1}$ and $\tau_{2}$ represent the initial pulse widths. It should be emphasized that the chirped solitons obtained by this method has already been correlated with the chirped solitons derived by the self-similar scaling analysis $[3,4,5]$. Based on this fact, the chirped two-soliton may imitate the self-similar property during the course of propagation along the exponentially decreasing dispersive media. This chirped two-soliton also enhances the chirp and maintains it during the propagation by establishing the balance between the exponentially growing chirp with that of the exponentially decreasing chirp induced by the exponentially decreasing dispersion.

\section{High Degree of Pulse Compression}

As has been discussed earlier, the main objective of the paper is to explore the high degree pulse compression through the special soliton pulse whose amplitude is twice of that of the fundamental soliton. The most important and interesting special case which occurs at $z$ $=0$, when the eigenvalues are 1 and 3 respectively. Under these parametric conditions, the general twosoliton solution becomes a familiar hyperbolic secant shape whose amplitude is doubled:

$$
\begin{aligned}
& \Psi(z=0)=\frac{2}{T_{0}} \sqrt{\frac{\beta_{0}}{\gamma}} \operatorname{sech}\left(t / T_{0}\right) \exp \left(i \frac{\alpha_{0}}{2} t^{2}+i \frac{\pi}{8}\right), \\
& \alpha_{0}=-4 / T_{0}^{2} / \pi .
\end{aligned}
$$

The above chirped soliton breather differs from the conventional breather mainly because of the initial chirp associated with the special 'sech' type pulse. This is the simplest example of a higher order soliton $(N=2)$, where the amplitude is doubled compared to that of a fundamental soliton. This kind of initially chirped higher order soliton breather enjoys advantage over the fundamental soliton in optical communication systems, as it undergoes extremely high degree pulse compression. We have used the split step fourier method to study the chirped two soliton breather, and the simulation results agree with the analytic solution very well. Fig. 1 shows the evolution of chirped two soliton breather in the expoentially decreasing dispersion. The physical parameters are $T_{0}=10 \mathrm{ps}, \beta_{0}=-20 \mathrm{ps}^{2} / \mathrm{km}$, $\beta(z)=\beta_{0} \exp \left(-\alpha_{0} \beta_{0} z\right), \gamma=2 \mathrm{~W}^{-1} / \mathrm{km}$ and $L=12 \mathrm{~km}$. Fig. 2 (a) and (b) show the evolution of peak power and compression factor for Fig.1, where the solid curve and dashed curve represent that for the chirped two soliton breather and chirped one soliton, respectively. The period of the chirped two soliton breather is found to be $z_{0}=\ln [1+1 /(2 k+1)] / \alpha_{0} / \beta_{0}$, where $k=1,2,3 \ldots$ The 2 sech pattern is repeated over each section of length $z_{0}$, where $z_{0}$ decreases with the propagation length.

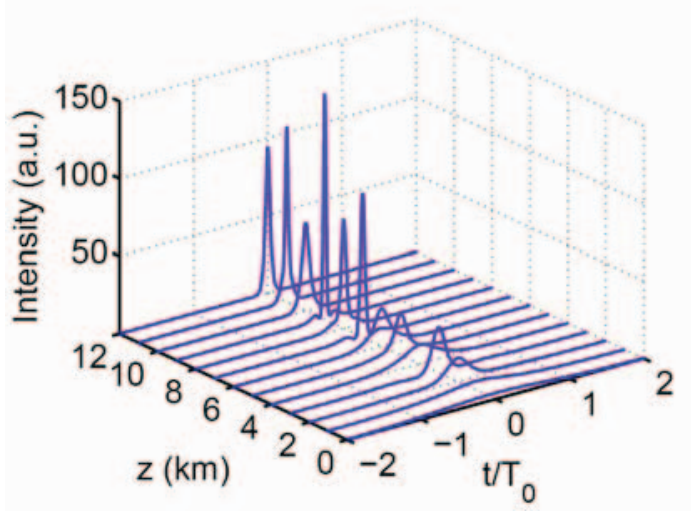

Fig.1. Compression of chirped soliton breather.

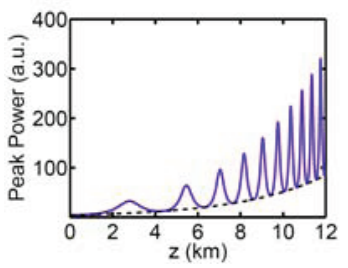

(a)

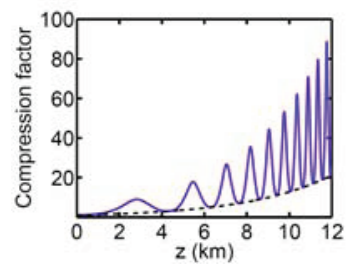

(b)
Fig.2. (a) Peak power and (b) compression factor of the chirped two soliton breather in Fig.1. The solid curve and dashed curve represent that for chirped two soliton breather and chirped one soliton, respectively.

\section{Conclusion}

The formation and generation of chirped higher order optical soliton has been discussed in the exponentially dispersion decreasing media. High degree pulse compressor has been proposed based on the the chirped soliton breather pulse.

\section{References}

1. G. P. Agrawal, Applications of Nonlinear Fiber Optics (Second Edition, Academic Press, New York, 2001).

2. J. D. Morres, Opt. Lett. 21, 55(1996).

3. V. I. Kruglov et al, Phys. Rev. E 71, 056619(2005).

4. Qian Li et al, J. Opt. Soc. Am. B 26, 432(2009).

5. K. Senthilnathan et al, "Chirped Optical Solitons" in "Advances in Nonlinear Waves and Symbolic Computation” (Nova, 2009). 